\title{
Torsten Verrel
}

\section{"When the green flag drops, the bullshit stops"}

\section{Anmerkungen zum Gesetz zur „Erweiterung der jugendgerichtlichen Handlungsmöglichkeiten“}

\section{Einleitung}

Am 4. September 2012 ist das vom Bundestag in seiner Sitzung vom 14. Juni mit den Stimmen der Regierungskoalition beschlossene Gesetz zur Erweiterung der jugendgerichtlichen Handlungsmöglichkeiten ${ }^{1}$ verkündet worden, das drei bedeutsame Änderungen des jugendstrafrechtlichen Sanktionsinstrumentariums enthält. Während die Schaffung einer gesetzlichen Grundlage für das richterrechtlich entwickelte Institut der sog. Vorbewährung - die Entscheidung über die Aussetzung einer durch Urteil verhängten Jugendstrafe wird erst einige Zeit nach der Urteilsverkündung im Beschlusswege ( $(57$ JGG) getroffen - uneingeschränkt zu begrüßen ist und hier nicht weiter kommentiert wird, erfolgte die Einführung des sog. Warnschussarrests ( $\$ 16 \mathrm{a}$ JGG), also der bislang unzulässigen Koppelung (vgl. $\ 8$ Abs. 2 a.F. JGG) von ausgesetzter Jugendstrafe (\$\$ 21, 27 JGG) mit Jugendarrest (\$16 JGG) sowie die Heraufsetzung der Jugendhöchststrafe für schwere Mordtaten Heranwachsender von 10 auf 15 Jahre ( $\mathbb{1 0 5}$ Abs. 3 Satz 2 JGG) gegen nahezu einhellig und seit langer Zeit von der Jugendstrafrechtswissenschaft geäußerte Bedenken. Auch wenn der Verf. zu den Kritikern gehört, ${ }^{2}$ soll der folgende Beitrag keine weitere Runde in dem für beinahe alle strafrechtlichen Reformen der jüngeren Vergangenheit typischen Aufeinanderprallen von Wissenschaft und einer deutlich repressiver gewordenen Kriminalpolitik einläuten. Denn einerseits hat der Gesetzgeber durchaus versucht, den Einwänden Rechnung zu tragen, die gegenüber dem Warnschussarrest - für den im Folgenden die sachlichere Bezeichnung „Koppelungsarrest“

* Alte Rennfahrerweisheit.

1 BGBl. I 2012, 1854; zu den parlamentarischen Vorgängen s. http://dipbt.bundestag.de/extrakt/ ba/WP17/442/44235.html; die Stellungnahmen der am 23.05.2012 öffentlich angehörten Sachverständigen finden sich unter http://webarchiv.bundestag.de/cgi/show.php?fileToLoad=2398\&id=1193.

2 Verrel/Käufl, NStZ 2008, 177; Verrel, Bonner Rechtsjournal 2/2008, $41 \mathrm{f}$.

NK 25. Jg. $1 / 2013$ 
verwendet wird ${ }^{3}$ - und den bisherigen Gesetzesinitiativen ${ }^{4}$ erhoben wurden. Zum anderen kann nun, da der Koppelungsarrest Gesetz geworden ist, vergleichsweise einfach überprüft werden, ob sich die mit der Einführung dieser Sanktionsform verbundenen Erwartungen oder Befürchtungen bestätigen. Dies gilt mit Einschränkungen auch für die Anhebung der Jugendhöchststrafe.

\section{Einführung von Koppelungsarrest}

\section{Pro und Contra}

Die zentralen Argumente der Befürworter und Gegner liegen seit Jahren auf dem Tisch. ${ }^{5}$ Es sind dies auf der einen Seite die Sorge vor der mangelnden Eindruckswirkung und Unrechtsverdeutlichung durch eine als solche nicht spürbare Bewährungsstrafe, zumal dann, wenn Mitangeklagte mit dem formal weniger schwerwiegenden, aber zu Freiheitsentzug führenden Jugendarrest sanktioniert werden, weiterhin die Ermöglichung des stationären Zugriffs auf gefährdete Jugendliche als gleichsam empfänglich machende Initialmaßnahme für die Bewährungshilfe und schließlich die Erwartung, dass die zusätzliche Option des Koppelungsarrests zu mehr positiven Legalprognosen und damit ausgesetzten Jugendstrafen führen wird.

Demgegenüber stehen die durch die Rückfallstatistik ${ }^{6}$ und Arrestantenbefragungen ${ }^{7}$ genährte Skepsis gegenüber einer nachhaltigen Abschreckungswirkung des „taste of prison", der Hinweis auf die bei mit Jugendstrafe belegten Jugendlichen vielfach bereits vorhandene Hafterfahrung und auf die schon de lege lata bestehenden Möglichkeiten, Bewährungsstrafen durch Auflagen, Weisungen und Belehrungen spürbar zu machen und schließlich die Befürchtung, dass der Koppelungsarrest nicht auf denkbare Sonderfälle beschränkt bleibt, sondern sich zu einer Routinesanktion mit absehbaren Konsequenzen für den schon jetzt überforderten Jugendarrestvollzug entwickelt.

3 Zur Problematik dieses Begriffs s. Verrel/Käufl (o. Fn. 2), 178; Müller-Piepenkötter/Kubink, ZRP 2008, 177 schlagen die Bezeichnung „Warnarrest“ vor; neutral wäre außerdem der Terminus „Bewährungsarrest“, während „Einstiegsarrest“ als Auftakt für weitere stationäre Sanktionen missverstanden werden könnte.

4 S. BT-Dr. 14/3189; BT-Dr 15/3422; BT-Dr 16/1027.

5 S. außer Verrel/Käufl (o. Fn. 2) und Müller-Piepenkötter/Kubink (o. Fn. 3) u.a. Findeisen ZJJ 2007, 25, 30; Breymann/Sonnen, NStZ 2005, 669, 673; Vietze, Der Einstiegsarrest - eine zeitgemäße Sanktion? 2004; Werwigk-Hertneck/Rebmann, ZRP 2003, 229 f. Jüngst kritisch: Kreuzer, ZRP 2012, 101.

6 Nach der im Jahr 2010 vom Bundesministerium der Justiz herausgegebenen bundesweiten Rückfalluntersuchung vonJeble/Albrecht/Hohmann-Fricke/Tetal hat derJugendarrest nach der unbedingten Jugendstrafe die zweithöchste Rückfallrate mit fast $65 \%$ (S. 60).

7 Zuletzt mit größeren Fallzahlen Schwegler, KrimJ 2001, 116, 126 f. 


\section{Konturierungsbemühungen des Gesetzgebers}

Der Gesetzgeber war sich der Problematik durchaus bewusst, dass dem Koppelungsarrest in dem schon jetzt weit ausdifferenzierten, ambulanten Maßnahmen den Vorzug einräumenden jugendstrafrechtlichen Sanktionensystem nur ein schmaler Anwendungsbereich zukommen kann und darf. Denn die zentrale Vorschrift des \16a JGG mit ihren drei, sogleich näher darzustellenden Arrestvarianten und mehr noch deren Erläuterung in der Gesetzesbegründung verfolgen erkennbar das Ziel eines sparsamen Einsatzes des Koppelungsarrests. Insoweit hat die Kritik aus dem Schrifttum sehr wohl Einfluss auf das Gesetzgebungsverfahren gehabt. Dies verdient ungeachtet aller grundsätzlichen und auch nach dem Gesetz verbleibenden Bedenken Respekt und Anerkennung. Bei der Lektüre der erfreulich ausführlichen und fachkundigen Begründung des Gesetzentwurfs $^{8}$ ist freilich die Schwierigkeit der Aufgabe ihrer Verfasser zu spüren, die deutliche Verschärfung des Jugendstrafrechts trotz der seit 2008 rückläufigen Entwicklung der Jugend(gewalt)kriminalität ${ }^{9}$ und trotz des betonten Vorrangs der Prävention vor der Repression zu rechtfertigen. Dies drückt sich auch darin aus, dass in der Begründung strenge Anforderungen an die in allen drei Varianten vorzunehmende Eignungs- und Erforderlichkeitsprüfung gestellt werden, ${ }^{10}$ die offensichtlich an die Stelle einer möglichen, noch engeren Fassung des Gesetzes getreten sind. Es wird sich zeigen, wie die Praxis mit dieser Diskrepanz umgeht.

\section{Die drei Arrestvarianten des $\int 16 a J G G$}

\section{a) „Verdeutlichungsarrest“}

Mit $\ 16 a$ Abs. 1 Nr. 1 JGG sollen die den Befürwortern des Koppelungsarrests in erster Linie vorschwebenden Fälle solcher Jugendlichen erfasst werden, die eine zur Bewährung ausgesetzte Jugendstrafe nicht ernst nehmen, sondern - so die immer wieder zu hörende Beschreibung - als "Freispruch zweiter Klasse" missverstehen. Ob der Jugendarrest „geboten ist, um dem Jugendlichen seine Verantwortlichkeit für das begangene Unrecht und die Folgen weiterer Straftaten zu verdeutlichen“, muss „unter Berücksichtigung der Belehrung über die Bedeutung der Aussetzung zur Bewährung und unter Berücksichtigung der Möglichkeit von Weisungen und Auflagen" beurteilt werden.

Flankiert wird diese Einschränkung, die angesichts der schon bisher bestehenden Möglichkeiten, Bewährungsstrafen durch Begleitmaßnahmen fühlbar zu machen, zu Recht in das Gesetz aufgenommen wurde, von einer neuen Vorschrift zur Gestaltung von Belehrungen, $\$ 70$ a JGG. Dessen Abs. 1 verlangt für alle Belehrungen - und eigentlich selbstverständlich - eine dem „Entwicklungs- und Bildungsstand“ des Jugendlichen ent-

8 BT-Drs. 17/9389, abrufbar unter http://dipbt.bundestag.de/dip21/btd/17/093/1709389.pdf.

9 Der seinerzeitige Anstieg der registrierten Jugendstraftaten war für Müller-Piepenkötter/Kubink, (o. Fn. 3), 176 f. ein zentrales Argument für die Einführung eines Warnschussarrests.

10 Gesetzentwurf(o. Fn. 8), S. 12 f.

NK 25. Jg. 1/2013 
sprechende Gestaltung. In Abs. 2 wird auf die besondere Situation eingegangen, dass neben dem zu ausgesetzter Jugendstrafe verurteilten und entsprechend belehrten Jugendlichen Mitangeklagte anwesend sind, die nur zu Erziehungsmaßregeln und Zuchtmitteln verurteilt werden. Diese Konstellation wurde, wie bereits oben erwähnt, als Argument für den Koppelungsarrest angeführt, mit dem man dem Eindruck entgegentreten könne, dass der zu der schwereren, aber eben ausgesetzten Jugendstrafe verurteilte Jugendliche milder bestraft werde als Mitangeklagte, die nur mit Jugendarrest, aber eben mit einer sogleich freiheitsentziehenden Sanktion belegt wurden. Zutreffend weist die Gesetzesbegründung darauf hin, dass bloße Gerechtigkeitserwägungen keine Korrektur dieses vermeintlichen Missverhältnisses legitimieren können. ${ }^{11}$ Jugendstrafrechtliche Sanktionen dienen eben nicht, jedenfalls nicht in erster Linie dem proportionalen Schuldausgleich, ${ }^{12}$ sondern richten sich nach den individuellen erzieherischen Bedürfnissen. Damit ist allerdings auch das Einfallstor für jugendstrafrechtsfremde Erwägungen bzw. der mögliche Etikettenschwindel in solchen Komplizenfällen offensichtlich, bereitet es doch keine allzu großen Mühen, auf den negativen Eindruck abzustellen, den spezialpräventiv wohl begründete Strafzumessungsunterschiede bei mehreren Tatbeteiligten auf den mit „nur“ ausgesetzter Jugendstrafe belegten Haupttäter haben könnten. Man wird daher sehr genau hinschauen müssen, wie ernst die Jugendgerichte die sich aus den $\int \mathbb{S} 16 \mathrm{a}$ Abs. 1 Nr. 1, 70a Abs. 2 JGG ergebenden Begründungsanforderungen an einen Verdeutlichungsarrest nehmen.

Eine weitere, unbedingt notwendige Einschränkung dieser Arrestvariante enthält $\$ 16 a$ Abs. 2 JGG. Da Freiheitsentzug die ihm zugeschriebene Schockwirkung nur dann in vollem Umfang haben kann, wenn er eine neue Erfahrung darstellt, wird in dieser Vorschrift die Regelvermutung mangelnder Gebotenheit eines Verdeutlichungsarrests bei solchen Jugendlichen ausgesprochen, die sich bereits früher im Jugendarrest oder in U-Haft befunden haben. Warum diese Negativvoraussetzung allerdings von vornherein auf Vorerfahrungen mit Dauerarrest und nicht nur kurzfristiger U-Haft beschränkt ist, leuchtet nicht ein. Auch und gerade ${ }^{13}$ in erstmaligen Fällen von Kurz- und Freizeitarrest oder wenige Stunden/Tage dauernder U-Haft kann der Freiheitsentzug sehr intensiv empfunden werden, seine Wirkung dann aber im Wiederholungsfall ebenso verlieren. Ausnahmen von der Vermutung, dass auch kürzere Freiheitsentziehungen den Verdeutlichungseffekt eines Koppelungsarrests konterkarieren, hätten ohne Weiteres berücksichtigt werden können, da $\$ 16 a$ Abs. 2 JGG ja nur für den Regelfall gilt.

b) „Herausnahmearrest“

Eine andere Zielsetzung als die der Unrechtsverdeutlichung durch Freiheitsentzug verfolgt die in $\$ 16 a$ Abs. 1 Nr. 2 JGG geregelte zweite Variante, die man auch als „Isolierungsarrest“ bezeichnen könnte. Sie ist dazu gedacht, Jugendliche „zunächst für eine

11 Gesetzentwurf (o. Fn. 8), S. 13.

12 Eine Ausnahme stellt die Jugendstrafe wegen Schwere der Schuld dar, $\$ 17$ Abs. 2. Alt. 2 JGG.

13 Vgl. Verrel/Käufl (o. Fn. 2), 179. 
begrenzte Zeit aus einem Lebensumfeld mit schädlichen Einflüssen herauszunehmen und durch die Behandlung im Vollzug des Jugendarrests auf die Bewährungszeit vorzubereiten." Aus diesem durchaus berechtigten Anliegen ergeben sich allerdings besondere Begründungsanforderungen. Denn so sinnvoll die Unterbrechung kriminogener Kontakte durch Inhaftierung als Initialmaßnahme für die anschließende ambulante Behandlung von durch ihr Umfeld gefährdeten Jugendlichen sein kann, so sehr hängt die Berechtigung einer derart motivierten Arrestierung davon ab, dass tatsächlich eine konkrete Maßnahmenperspektive sowohl für die weitere Arrestdauer, als auch und vor allem für die Zeit danach besteht. Gerade bei dieser Zielgruppe wäre ein Vertrauen auf die läuternde Wirkung allein schon des Vollzugserlebnisses naiv. Denn wenn sich die Pforten der Arrestanstalt für die Jugendlichen nach wenigen Tagen, maximal vier Wochen, wieder öffnen, sind sie den schädlichen Bedingungen und Einflüssen ihres Milieus erneut ausgesetzt und dürfte sich in so kurzer Zeit auch wenig an ihrer bislang offenbar fehlenden Fähigkeit geändert haben, diesen widrigen Bedingungen zu trotzen. Hier stößt der Jugendarrest in allen seinen gegenwärtigen Erscheinungsformen an Grenzen und zeigt eine Schwachstelle des jugendstrafrechtlichen Sanktionensystems insgesamt auf: Es kennt unterhalb der Schwelle einer vollstreckten Jugendstrafe kein geeignetes (halb)stationäres, gleichwohl erzieherisch ausgestaltetes Reaktionsmittel für mehrfach auffällige und nach ihren Lebensumständen weiterhin gefährdete Jugendliche.

Doch zurück zu $\ 16$ Abs. 1 Nr. 2 JGG. Die Einsicht in die auch in der Gesetzesbegründung eingeräumte Wirkungslosigkeit des schlichten Einsperrens bei gefährdeten Jugendlichen führt zu der darin formulierten Einschränkung, dass Herausnahmearrest nur dann verhängt werden kann, ,wenn eine (...) Behandlung im Arrestvollzug tatsächlich zu erwarten ist und dieser sich nicht lediglich auf den Freiheitsentzug und die vorübergehende Isolierung zum Beispiel von einer delinquenzgeneigten Gleichaltrigengruppe beschränkt. " ${ }^{14}$ Auch für die Zeit danach findet sich in der Gesetzesbegründung der bemerkenswerte und mit allem Nachdruck zu betonende Hinweis, dass „eine nachhaltige Wirkung (...) im Übrigen in der Regel voraussetzen (wird), dass eine geeignete und angemessene Übergangs- und Nachbetreuung durch die Bewährungs- und/oder die Jugendgerichtshilfe im Anschluss an den Arrestvollzug sichergestellt ist." Da diese notwendigen Einschränkungen leider keinen Eingang in den Gesetzeswortlaut gefunden haben, der nur ein nicht weiter konkretisiertes Gebotensein verlangt, bleibt wiederum abzuwarten, wie die Praxis mit dem Herausnahmearrest umgeht. Nimmt sie seine Zielsetzung und die sich daraus ergebenden Begründungsanforderungen ernst und finden sich engagierte Jugendrichter, die auf die Erarbeitung von Nachhaltigkeitskonzepten drängen, könnten sich aus dieser Variante des Koppelungsarrests und ganz in der Tradition der das Jugendstrafrecht prägenden „Reform von unten “15 wertvolle Impulse für die Fortentwicklung des Jugendarrests insgesamt ergeben. ${ }^{16}$

14 Gesetzentwurf (o. Fn. 8), S. 13.

15 S. dazu Schöch, FS-Brunner 2001, $125 \mathrm{ff} .$.

16 Als Beispiel für derartige Ansätze kann das von Benninghoff-Giese/Wessiepe, FS 2012, $99 \mathrm{f}$. beschriebene Modellprojekt der JAA Wetter für junge Frauen dienen, dessen harte Ergebnisevaluation freilich noch aussteht.

NK 25. Jg. $1 / 2013$ 
c) „Auffangarrest“

Konnte dem Gesetzgeber bisher bescheinigt werden, sich um eine Eingrenzung der Fälle des Koppelungsarrests bemüht zu haben, wird dieses Bild durch den in $\$ 16$ Abs. 1 Nr. 3 geregelten dritten Anwendungsfall nachhaltig getrübt. Danach kommt Jugendarrest auch in Betracht, wenn „dies geboten ist, um eine nachdrücklichere erzieherische Einwirkung (...) zu erreichen oder (...) bessere Erfolgsaussichten für eine erzieherische Einwirkung in der Bewährungszeit zu schaffen." Diese beiden Varianten sind letztlich eine allgemeiner formulierte Umschreibung der in den Nrn. 1 und 2 geregelten Fälle, so dass unklar bleibt, welche Konstellationen mit der Nr. 3 aufgefangen werden sollen. Nicht ohne Grund findet sich in der Gesetzesbegründung hierfür kein konkretes Fallbeispiel, wohl aber der Hinweis, dass sich die für beide Konstellationen erforderliche Eignung zur nicht nur unwesentlichen Verbesserung der Legalbewährungsaussichten „aus konkret festzustellenden Umständen ergeben (muss)“, die „vom Gericht gegebenenfalls in den Urteilsgründen dargelegt werden (müssen). "17 Somit besteht für die Nr. 3 ein nochmals erhöhter Begründungsbedarf, gilt es aber wiederum zu überprüfen, ob diese „Begründungslösung“ in der Rechtspraxis tatsächlich zur Konturschärfung der dritten Variante und zur Einsicht in eine Bandbreite sinnvoller Koppelungsarreste führt oder von den Gerichten einfach ignoriert wird wie dies beispielsweise aus dem Umgang mit $₫ 3$ JGG bekannt ist, ${ }^{18}$ der sogar eine gesetzliche Begründungspflicht enthält.

\section{Arrestvollstreckung}

Positiv zu bewerten ist dagegen das für alle Varianten geltende, in $\ 87$ Abs. 4 Satz 2 JGG aufgenommene verschärfte Vollstreckungsverbot nach Ablauf von drei Monaten seit Rechtskraft, das sich deutlich von der - viel zu langen - Jahresfrist beim normalen Jugendarrest (Satz 1) abhebt, allerdings durchaus noch kürzer hätte bemessen werden können. ${ }^{19}$ Wenn der Jugendarrest die erhoffte motivierende Wirkung für die Bewährungszeit entfalten soll, muss er unbedingt an deren Anfang stehen. ${ }^{20}$ Es wäre geradezu fatal, wenn er mit ambulanten Maßnahmen der Bewährungshilfe oder gar einem begonnenen Ausbildungsverhältnis kollidieren würde. Die Entwurfsbegründung erwähnt in diesem $\mathrm{Zu}$ sammenhang in bemerkenswerter Offenheit die „kaum vermeidbaren schädlichen Nebenwirkungen des Vollzugs" ${ }^{21}$ die allerdings auch bei zeitnaher Arrestvollstreckung bestehen und damit schon bei der Anordnung von Koppelungsarrest, insbesondere in der Variante des Verdeutlichungsarrests zu Zurückhaltung mahnen sollten.

17 Gesetzentwurf (o. Fn. 8), S. 13.

18 S. nur Ostendorf, JGG Kommentar, 9. Aufl. 2013, Grdl. z. \$3 Rn 4.

19 Müller-Piepenkötter/Kubink (o. Fn. 3), 179 sahen eine Höchstfrist von einem Monat als eine der „kriminologische(n) Grundbedingungen eines Erfolg versprechenden Modells eines Warnarrests" an.

20 Insoweit ist die früher gebräuchliche Bezeichnung „Einstiegsarrest“ zutreffend.

21 Gesetzentwurf (o. Fn. 8), S. 19. 
$\mathrm{Ob}$ das in dem Vollstreckungsverbot zum Ausdruck kommende, für das gesamte Jugendstrafrecht bisher so einhellig betonte Ideal einer tatzeitnahen Sanktionierung tatsächlich die ihr ohne weiteres zugeschriebene Überlegenheit gegenüber späteren Sanktionen hat, sei hier dahingestellt. Insoweit steht nämlich noch sowohl ein empirischer als auch genauer lerntheoretischer Nachweis aus. ${ }^{22}$ \$ 87 Abs. 4 Satz 2 JGG hat jedenfalls seine Berechtigung darin, Maßnahmenkollisionen durch Zeitverzug zu verhindern sowie die besonders im Jugendalter jederzeit möglichen Wendepunkte und kurzfristigen Stabilisierungen nicht zu konterkarieren. Gilt es daher, auch über $\ 87$ Abs. 3 Satz 1 JGG (Vollstreckungsverzicht aus zwischenzeitlich hervorgetretenen erzieherischen Gründen) hinaus Raum für eine Bewährung ohne Arrestvollzug zu lassen, macht der Vollzug von Koppelungsarrest aber ebenso wenig in den umgekehrten, nunmehr in Satz 3 in Bezug genommenen Fällen einer widerrufenen Strafaussetzung ( $\$ 26$ JGG), nach $\ 30$ Abs. 1 JGG verhängten unbedingten Jugendstrafe oder einer nach misslungener Vorbewährung abgelehnten Strafaussetzung (\$61a Abs. 1 JGG) Sinn. ${ }^{23}$

Abgesehen davon, dass das verschärfte Vollstreckungsverbot eine zwingend erscheinende Konsequenz des hinter dem Koppelungsarrest stehenden Sanktionskonzepts ist, hat $\$ 87$ Abs. 4 Satz 2 JGG darüber hinaus eine ganz entscheidende Bedeutung für dessen praktische Umsetzung. Denn nunmehr besteht ein ganz erheblicher Druck, das Vollstreckungsverfahren zu beschleunigen und d.h. vor allem, eine ausreichende Zahl an Arrestplätzen bereit zu stellen, wenn man nicht riskieren möchte, dass Koppelungsarreste wegen Fristablaufs reihenweise nicht mehr vollstreckt werden dürfen. Damit ist zugleich der vermutlich wundeste Punkt des Gesetzes angesprochen: Ein nicht nur auf minimale Fallzahlen beschränkter Koppelungsarrest kann auch bei noch so guter Ausgestaltung seiner gesetzlichen Voraussetzungen überhaupt nur funktionieren, wenn die Bundesländer in ihren Arrestvollzug investieren und die ebenfalls in ihre Zuständigkeit fallenden Vollstreckungsabläufe optimieren. Die Erweiterung der jugendgerichtlichen Reaktionsmöglichkeiten um den Koppelungsarrest ist mit anderen Worten ein totgeborenes Kind, wenn die Länder nicht ihren Teil auf der Vollstreckungs- und vor allem Vollzugsseite mittun. ${ }^{24}$

\section{Auswirkungen auf die Prognosestellung nach \21 JGG}

In der Gesetzesbegründung wird zu diesem Erfüllungsaufwand die vorsichtige Gegenrechnung aufgemacht, dass der Vollzug von Koppelungsarrest auch zu Einsparungen durch eine verringerte Zahl vollstreckter Jugendstrafen führen kann. ${ }^{25}$ Dies wäre zum

22 S. dazu Verrel, FS Heinz 2012, 521521, 527 ff.; auf einen sogar umgekehrten Zusammenhang deuten die Ergebnisse von Bliesener/Thomas, ZJJ 2012, 382 ff. hin.

23 Sofern das Bewährungsversagen wie meistens in einer erneuten Straftat besteht, eröffnete schon bisher $₫ 87$ Abs. 3 Satz 3 JGG eine allerdings im Ermessen des Vollstreckungsleiters liegende Verzichtsmöglichkeit.

$24 \mathrm{Zu}$ den derzeit defizitären erzieherischen Rahmenbedingungen des Jugendarrestsvollzugs in NRW s. Benninghoff-Giese/Wessiepe (o. Fn. 20), 99.

25 Gesetzentwurf (o. Fn. 8), S. 3, 10.

NK 25. Jg. 1/2013 
einen dann der Fall, wenn der Koppelungsarrest tatsächlich zu weniger Rückfällen innerhalb, aber auch nach dem Ende der Bewährungszeit führen würde. Zum anderen könnte eine Verringerung unbedingter Jugendstrafen dann eintreten, wenn die Jugendgerichte erst aufgrund der Option eines Koppelungsarrests zu einer günstigen Einschätzung der Legalbewährung kommen und daher häufiger als bisher Jugendstrafen zur Bewährung aussetzen würden. Diese Erwartung ist keineswegs von vornherein unbegründet, da die voraussichtlichen Wirkungen der eine ausgesetzte Jugendstrafe flankierenden $\mathrm{Maßnahmen}$ von entscheidender prognostischer Bedeutung sind. ${ }^{26}$

Der Gesetzgeber sah sich zur Vermeidung von Auslegungsproblemen ${ }^{27}$ dazu veranlasst, die maßgebliche Vorschrift des $\$ 21$ Abs. 1 JGG in einem neuen Satz 3 um den ausdrücklichen Hinweis zu ergänzen, dass eine Strafaussetzung auch dann zu erfolgen hat, wenn die Erwartung künftiger Straffreiheit „erst dadurch begründet wird, dass neben Jugendstrafe ein Jugendarrest nach $\$ 16 a$ verhängt wird. “ Das überzeugt zwar nicht, da unter die Formulierung in Satz 1, „dass der Jugendliche sich schon die Verurteilung zur Warnung dienen“ lässt „und (...) unter der erzieherischen Einwirkung in der Bewährungszeit künftig einen rechtschaffenen Lebenswandel führen wird,“ ohne allzu große Auslegungsnöte auch die prognostizierten Effekte eines Koppelungsarrests subsumiert werden können.

Sinnvoll ist diese Ergänzung gleichwohl, denn die Worte „erst dadurch“ machen im Umkehrschluss deutlich, dass der Koppelungsarrest nicht in solchen Fällen angeordnet werden darf, in denen man bisher schon, also ohne die Option einer Arrestverhängung, zu einer günstigen Beurteilung des künftigen Legalverhaltens gekommen ist. Ob damit allerdings die Gefahr eines net-widening-Effekts durch unnötige Koppelungsarrestdraufgaben wirklich gebannt werden kann, darf bezweifelt werden. Denn abgesehen davon, dass es nur eine Frage der in diesem Punkt kaum überprüfbaren Urteilsbegründung ist, ob das Gericht ohne Koppelungsarrest auszukommen glaubt oder darin das letzte Mosaiksteinchen zur Begründung einer positiven Legalprognose sieht, dürfte allein schon die Existenz dieser Aufrüstungsmöglichkeit von Bewährungsstrafen entscheidungspsychologische Bedeutung haben.

\section{Höchststrafenanbebung für Heranwachsende bei qualifiziertem Mord}

Verglichen mit der in der Vergangenheit erhobenen Forderung, die Jugendhöchststrafe für Heranwachsende generell von 10 auf 15 Jahre heraufzusetzen, ${ }^{28}$ handelt es sich bei der auf Mordtaten mit „besonderer Schwere der Schuld“ begrenzten Strafrahmenerhöhung um eine sehr eng umschriebene Verschärfung des Jugendstrafrechts, die nur in extremen Ausnahmefällen zum Tragen kommen kann. Denn es ist schwer vorstellbar, wie

26 Vgl. Rössner in: Meier/Rössner/Schöch, Jugendstrafrecht, 3. Aufl. 2013, $\$ 6$ Rn 44.

27 Gesetzentwurf (o. Fn. 8), S. 14.

28 So etwa der Gesetzentwurf der CDU/CSU-Bundestagsfraktion vom 12.04.2000 (BT-Drs. 14/3189); einschränkend auf „schwerste Verbrechen“ die Wiesbadener Erklärung der CDU vom 5.1.2008 (abrufbar unter http://www.cdu.de/doc/pdfc/080105-wiesbadender-erklaerung.pdf), S. 8. 
trotz der Annahme einer Entwicklungsretardierung nach $\ 105$ Abs. 1 Nr. 1 JGG - der Fall eines besonders schuldschweren, zugleich „jugendtümlichen“ Mordes nach Nr. 2 dürfte nicht vorkommen - noch Raum für die Erhebung eines qualifizierten, also über die Verwirklichung von Mordmerkmalen hinzugehenden Schuldvorwurfs sein soll, zumal sich nach der Rechtsprechung die Höhe einer Jugendstrafe wegen Schwere der Schuld vorrangig nach erzieherischen Gesichtspunkten zu richten hat. ${ }^{29}$ Sind von dieser Strafrahmenverschiebung als solcher folglich keine nachhaltigen Veränderungen der Strafzumessungspraxis zu erwarten, ${ }^{30}$ zeigt sie doch zum einen in aller Deutlichkeit auf, welche Spannungen entstehen, wenn in das präventiv ausgerichtete Jugendstrafrecht ausschließlich schuldvergeltende Elemente aus einem Problembereich des Erwachsenenstrafrechts importiert werden, in dem es um die vollstreckungsrechtliche, auf der Strafzumessungsebene derzeit nicht mögliche Ausdifferenzierung der lebenslangen Freiheitsstrafe geht. Denn mit der Formulierung „wegen der besonderen Schwere der Schuld“ wird die Schuldschwereklausel des $\ 57$ a Abs. 1 Nr. 2 StGB übernommen, deren Definition und Handhabung durch die Rspr. bekanntlich nicht unproblematisch ist ${ }^{31}$ und deren Existenz die Folge der Unzulänglichkeiten der absoluten Strafdrohung beim Mord $^{32}$ bzw. des Bedürfnisses ist, auch bei den höchststrafwürdigen Mordfällen Sanktionsschwereabstufungen vorzunehmen.

Zum anderen - und darin liegt die eigentliche, in der Entwurfsbegründung ${ }^{33}$ wiederum erfreulich offen angesprochene Problematik der Erweiterung des $\$ 105$ Abs. 3 JGG besteht die Gefahr, dass die Höchststrafenanhebung in Satz 2 auf die Strafzumessungspraxis innerhalb des Normalstrafrahmens von Satz 1 ausstrahlt. Die Neuregelung könnte nämlich als gesetzgeberisches Signal verstanden werden, dem Vergeltungsgedanken im Jugendstrafrecht fortan nicht nur bei qualifizierten Mordtaten, sondern auch in anderen Fällen schwerer, öffentlichkeitswirksamer Gewaltstraftaten Heranwachsender stärker Rechnung zu tragen. Die Jugendgerichte stehen heute erheblich unter medialem Druck, wenn Sie bei Aufsehen erregenden Gewaltdelikten heranwachsender Täter Jugendstrafrecht anwenden und sich bei der Sanktionierung konsequent am Erziehungsgedanken des JGG und damit nicht an den Straf- und Vergeltungserwartungen der Bevölkerung orientieren. In dieser Situation könnte das Wissen um die Existenz einer ausnahmsweisen Jugendhöchststrafe von 15 Jahren die Bereitschaft fördern, näher an die bisherige ausschließliche Höchststrafe von 10 Jahren heranzurücken.

29 BGH NStZ-RR 2012, 186; 2008, 258 f.; ZJJ 2003, 302.

30 Im Jahr 2011 wurden gerade einmal 9 Heranwachsende wegen vollendeten Mordes nach Jugendstrafrecht verurteilt (Strafverfolgungsstatistik 2011, Tab. 2.1, S. 33; abrufbar www.destatis.de -> Zahlen \& Fakten -> Rechtspflege -> Strafverfolgung).

31 S. nur Dünkel in NK-StGB, 3. Aufl. 2010, \$57a Rn 7 ff. mwN.

32 Näher dazu und mit Reformvorschlägen, Heine et al., Alternativ-Entwurf Leben, GA 2008, $193 \mathrm{ff}$.

33 Gesetzentwurf (o. Fn. 8), S. 20.

NK 25. Jg. $1 / 2013$ 


\section{Forschungsbedarf}

$\mathrm{Ob}$ es tatsächlich dazu kommt oder diese ebenso wie die anderen Erweiterungen der jugendgerichtlichen Handlungsmöglichkeiten von der Praxis mit Bedacht und Augenmaß eingesetzt werden, muss sich nun erweisen.

\section{Koppelungsarrest}

Die von der Jugendstrafrechtswissenschaft geäußerten Bedenken gegen dessen Einführung sind zweifellos gewichtig, doch darf nicht übersehen werden, dass es bisher keine wirklich harten Fakten zur Wirkung(slosigkeit) gerade der Kombination von Bewährungsstrafe und Jugendarrest gibt und der Gesetzgeber wie gesehen um eine Eingrenzung der Anwendungsfälle bemüht war. Jetzt besteht die Möglichkeit, aber auch die Notwendigkeit, im Feld zu überprüfen, welchen Platz der Koppelungsarrest in der jugendgerichtlichen Sanktionspraxis einnimmt und welchen Einfluss der "taste of prison“ auf den Bewährungserfolg hat.

Das Forschungsprogramm und die Forschungsmethode liegen damit weitgehend auf der Hand. Es wird in einem ersten deskriptiven Schritt darum gehen, mittels einer Aktenerhebung, die durch eine Richterbefragung flankiert werden könnte, Anzahl und Merkmale der Fälle zu erfassen, in denen Koppelungsarrest angeordnet wurde. Dies erlaubt bereits erste Aussagen darüber, welche Bedeutung, insbesondere Begrenzungsfunktion die drei Fallgruppen des $\$ 16 a$ Abs. 1 JGG haben. Besonderes Augenmerk ist dabei auf die Ergiebigkeit der Urteilsbegründungen zu legen, mit denen die Gebotenheit des Koppelungsarrests namentlich in der diffus erscheinenden Variante des „Auffangarrests“ nach Nr. 3 dargelegt werden. In der Variante des „Verdeutlichungsarrests“ nach Nr. 1 ist vor allem die Handhabung der Regelvermutung des Abs. 2 zu beleuchten. Um die Auswirkungen des Koppelungsarrests auf die Aussetzungsbereitschaft der Jugendgerichte zu überprüfen, muss die Entwicklung des Verhältnisses von unbedingten und bedingten Jugendstrafen mit einbezogen werden bzw. darauf geachtet werden, ob es bei einem gleich gebliebenen Anteil ausgesetzter Jugendstrafen zu möglicherweise nur draufgesattelten Jugendarresten gekommen ist. Da regional erheblich voneinander abweichendes Sanktionsverhalten gerade im Jugendstrafrecht nicht ungewöhnlich, aber durchaus problematisch ist - man denke etwa an die ausgeprägte Rechtszersplitterung im Bereich der Diversion ${ }^{34}$ - sollte unbedingt eine (ggf. stichprobenartige) bundesweite Erhebung der Praxis des Koppelungsarrests erfolgen.

In einem zweiten Schritt und als entscheidende Nagelprobe des (vollstreckten) Koppelungsarrests muss die Rückfallhäufigkeit untersucht werden. Als Vergleichsgruppe kommen zu Bewährungsstrafen nur mit den herkömmlichen Auflagen und Weisungen verurteilte Jugendliche/Heranwachsende mit gematchten Tat- und Tätermerkmalen entweder aus der Zeit vor Inkrafttreten des Erweiterungsgesetzes oder - bei entsprechend sparsamen Gebrauch des Koppelungsarrests - auch danach in Betracht. Der Rückfall-

34 S. dazu Verrel, FS Schöch 2010, 227, $236 \mathrm{ff}$.

THEMEN 
zeitraum könnte angesichts der erhofften kurz- und mittelfristigen Wirkungen des Koppelungsarrests, aber auch wegen der Dynamik der Persönlichkeitsentwicklung bei jungen Tätern und in Anlehnung an $\$ 28$ Abs. 1 JGG auf zwei Jahre begrenzt werden, so dass schon bald erste Aussagen über die Effizienz des Koppelungsarrests möglich wären.

Drittes Kernstück einer Evaluation sollten die vollstreckungsrechtlichen Aspekte und vollzuglichen Auswirkungen des Koppelungsarrests sein. Hier geht es vor allem um die Dauer zwischen Rechtskrafteintritt und Vollstreckungsbeginn, insbesondere um die Häufigkeit der wegen Überschreitens der Drei-Monatsfrist des $\ 87$ Abs. 4 JGG unzulässig gewordenen Arrestvollstreckungen. Daneben muss ermittelt werden, wie sich die Arrestanstalten auf die zusätzliche Klientel der Koppelungsarrestanten einstellen, ob es (nur) zu einer Kapazitätserweiterung oder (auch) zur Entwicklung von speziellen Behandlungs- und Nachsorgekonzepten und insbesondere zu der erforderlichen Verzahnung von Arrest und Folgemaßnahmen kommt.

\section{Höchststrafenanbebung}

Lassen sich demnach in einem überschaubaren Zeitraum Erkenntnisse darüber gewinnen, wie der Koppelungsarrest von den Jugendgerichten angenommen wird und welche Wirkungen er entfaltet, gestaltet sich die Untersuchung möglicher Ausstrahlungen der Strafrahmenerhöhung in $\$ 105$ Abs. 3 Satz 2 JGG auf die Strafzumessungspraxis in anderen Fällen schwerer Heranwachsendenkriminalität weitaus schwieriger. Die kontinuierliche Auswertung der Strafverfolgungsstatistik kann darüber keinen gesicherten Aufschluss geben, da die Strafhöhen in dem hier interessierenden Bereich in nur zwei weit gespannten Strafmaßgruppen ( 3 bis 5 und 5 bis 10 Jahre) erfasst werden, die ohnehin nur vergleichsweise geringe Besetzungszahlen aufweisen. ${ }^{35}$ Vor allem aber enthält die Strafverfolgungsstatistik über die bloße Nennung des der Verurteilung zugrunde liegenden Straftatbestands hinaus keine Angaben zur Ausprägung der Tat und fast keine Daten zum Täter, so dass auch nicht kontrolliert werden kann, ob ein etwaiger Anstieg des Anteils längerer Jugendstrafen die Folge einer veränderten Deliktsschwere bzw. schwierigerer Täterpersönlichkeiten und nicht einer schärferen Gangart der Jugendgerichte ist. ${ }^{36}$ Notwendig wäre daher wiederum eine strafaktenbasierte, einen längeren Zeitraum von Vorher- und Nachherjahrgängen umfassende Analyse solcher Fälle, in denen wegen Totschlags, Vergewaltigung und sexueller Nötigung, Körperverletzung mit Todesfolge, anderer qualifizierter Körperverletzungen oder wegen schweren Raubes unbedingte Jugendstrafen verhängt wurden. Angesichts des damit verbundenen Aufwands wird man sich einstweilen wohl damit begnügen müssen, die unmittelbare Bedeutung des $\$ 105$

35 Die Strafverfolgungsstatistik (o. Fn. 34), S. 296 f., Tab 4.2.2, weist für das Jahr 2011 insgesamt 10.458 Verurteilungen von Heranwachsenden zu Jugendstrafen wegen einer Straftat ohne Straßenverkehrsdelikte aus, von denen nur $97(0,9 \%) 5$ bis 10 Jahre und $501(4,8 \%) 3$ bis 5 Jahre betrugen.

36 Vgl. Heinz, Folienpräsentation: „Punitivität in der jugendstrafrechtlichen Sanktionierungspraxis!? Die Punitivitätsthese auf dem Prüfstand“, abrufbar unter http://www.uni-konstanz.de/rtf/kis/Heinz_2012_Punitivitaet_JGG.pdf.

NK 25. Jg. 1/2013 
Abs. 3 S. 2 JGG zu beobachten, und das heißt letztendlich abzuwarten, bis der BGH Gelegenheit erhält zu erläutern, wie die besondere Schwere der Schuld bei einem heranwachsenden, unter das Jugendstrafrecht fallenden Mörder auszulegen ist.

\section{Schluss}

Das bloße Spekulieren über die praktische Bedeutung von Höchststrafenanhebungen für Extremkriminalität, vor allem aber über Nutzen und Schaden des Koppelungsarrests kann und sollte jetzt ein Ende haben. Denn nun sind diese erweiterten Reaktionsmöglichkeiten in das Rennen der praktischen Anwendung gestartet und werden harte Fakten zeigen, ob sie die Ziellinie einer konstruktiven Ausdifferenzierung des jugendstrafrechtlichen Sanktionensystems erreicht haben oder das Rennen wegen der Zunahme bestenfalls unnötiger, schlimmstenfalls schädlicher Freiheitsentziehungen abgebrochen werden muss.

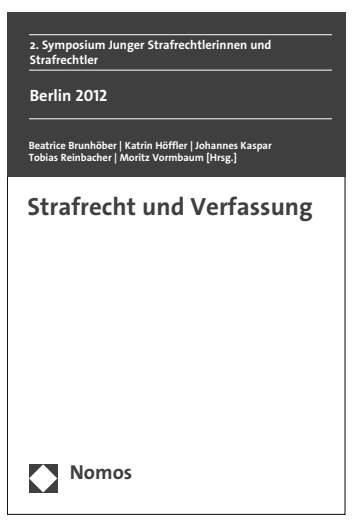

\section{Strafrecht und Verfassung}

2. Symposium Junger Strafrechtlerinnen und Strafrechtler

Berlin 2012

Herausgegeben von Dr. Beatrice Brunhöber, Jun.-Prof. Dr. Katrin Höffler, Prof. Dr. Johannes Kaspar, Dr. Tobias Reinbacher und Dr. Moritz Vormbaum

2013, 169 S., brosch., 44,- $€$

ISBN 978-3-8329-7905-8

Droht dem Strafrecht die „Kolonialisierung“ durch das Verfassungsrecht? Oder ist es eher ein „Freistaat“, der sich den Bindungen des Verfassungsrechts geradezu entzieht? Der Band enthält neben einem Vorwort von Prof. Dr. Dr. h.c. mult. Winfried Hassemer acht Beiträge junger Strafrechtswissenschaftlerinnen und -wissenschaftler zu grundlegenden Fragen aus dem Grenzbereich von Strafrecht und Verfassung. 